\title{
CD55 upregulation in astrocytes by statins as potential therapy for AQP4-lgG seropositive neuromyelitis optica
}

\author{
Lukmanee Tradtrantip ${ }^{1}$, Tianjiao Duan ${ }^{1,2}$, Michael R. Yeamann ${ }^{3,4}$ and Alan S. Verkman ${ }^{1 *}$
}

\begin{abstract}
Background: Neuromyelitis optica spectrum disorder (herein called NMO) is an inflammatory demyelinating disease that can be initiated by binding of immunoglobulin G autoantibodies (AQP4-lgG) to aquaporin-4 on astrocytes, causing complement-dependent cytotoxicity (CDC) and downstream inflammation. The increased NMO pathology in rodents deficient in complement regulator protein CD59 following passive transfer of AQP4-lgG has suggested the potential therapeutic utility of increasing the expression of complement regulator proteins.

Methods: A cell-based ELISA was developed to screen for pharmacological upregulators of endogenous CD55 and CD59 in a human astrocyte cell line. A statin identified from the screen was characterized in cell culture models and rodents for its action on complement regulator protein expression and its efficacy in models of seropositive NMO.

Results: Screening of 11,500 approved and investigational drugs and nutraceuticals identified transcriptional upregulators of CD55 but not of CD59. Several statins, including atorvastatin, simvastatin, lovastatin, and fluvastatin, increased CD55 protein expression in astrocytes, including primary cultures, by three- to four-fold at $24 \mathrm{~h}$, conferring significant protection against AQP4-IgG-induced CDC. Mechanistic studies revealed that CD55 upregulation involves inhibition of the geranylgeranyl transferase pathway rather than inhibition of cholesterol biosynthesis. Oral atorvastatin at $10-20 \mathrm{mg} / \mathrm{kg} /$ day for 3 days strongly increased CD55 immunofluorescence in mouse brain and spinal cord and reduced NMO pathology following intracerebral AQP4-IgG injection.
\end{abstract}

Conclusion: Atorvastatin or other statins may thus have therapeutic benefit in AQP4-IgG seropositive NMO by increasing CD55 expression, in addition to their previously described anti-inflammatory and immunomodulatory actions.

Keywords: NMO, Aquaporin-4, CD55, Astrocyte, Complement-dependent cytotoxicity, Statins

\section{Introduction}

Neuromyelitis optica spectrum disorder (herein called $\mathrm{NMO}$ ) is an inflammatory demyelinating disease of the central nervous system. In seropositive NMO, immunoglobulin $G$ autoantibodies against water channel aquaporin-4 (AQP4), called AQP4-IgG, bind to AQP4 on astrocytes and cause cytotoxicity largely by a complement-dependent mechanism [1-5]. Consequent inflammation, blood-brain barrier disruption, and oligodendrocyte injury result in demyelination and neurological deficit. Current therapy for NMO

\footnotetext{
* Correspondence: Alan.Verkman@ucsf.edu

'Departments of Medicine and Physiology, University of California, 1246

Health Sciences East Tower, 513 Parnassus Ave, San Francisco, CA

94143-0521, USA

Full list of author information is available at the end of the article
}

includes immunosuppressants, B cell depletion, and plasma exchange, and new therapeutics are in the development pipeline that target complement, cytokines, immune cells, and AQP4-IgG binding to astrocyte AQP4 [6-9].

Evidence for an important role of complement in seropositive NMO includes vasculocentric deposition of activated complement in NMO-affected human tissues [2, 1012], complement-dependent NMO pathology in rodents following passive transfer of AQP4-IgG [13-15], and efficacy of the C5 complement inhibitor eculizumab in a preliminary clinical trial [16]. Additional evidence includes the increased NMO pathology in mice and rats lacking complement regulator protein CD59 following passive transfer of AQP4-IgG into the central nervous system [15,

(c) The Author(s). 2019 Open Access This article is distributed under the terms of the Creative Commons Attribution 4.0 International License (http://creativecommons.org/licenses/by/4.0/), which permits unrestricted use, distribution, and 
17, 18]. NMO therapeutics that target components of the complement activation pathway can be associated with significant infectious and autoimmune side effects, as can agents that target B cells or other immune effectors [19, 20]. As an alternative approach, as motivated by the consequences of CD59 knockout [15, 17, 18], inhibition [15], and overexpression [21] in experimental animal models of $\mathrm{NMO}$, here we investigate the therapeutic utility of pharmacological upregulation of complement regulator proteins in astrocytes.

We report a high-throughput screen to identify small molecule transcriptional upregulators of CD55 and $\mathrm{CD} 59$, the major complement regulator proteins in astrocytes. For screening, a human astrocyte-derived cell line was identified that endogenously expressed low to moderate levels of CD55 and CD59. Compounds emerging from the screen included statins, which were characterized for their mechanism of action in increasing CD55 expression in astrocytes and for their potential therapeutic utility in NMO.

\section{Materials and methods Materials}

Purified recombinant AQP4-IgG (rAb-53, refs. [22, 23]) was provided by Dr. Jeffrey Bennett (Univ. Colorado, Denver). Human complement was purchased from Innovative Research (Novi, MI) and human (control) IgG from Pierce Biotechnology (Rockford, IL). GGTI-286 was purchased from Calbiochem (San Diego, CA), fasudil from Abcam (Cambridge, MA), and zaragozic acid A from Cayman Chemical Co. (Ann Arbor, MI). All other chemicals were purchased from Sigma-Aldrich (St. Louis, MO).

\section{Cell culture}

U-251MG (NCI) and U-373MG (ATCC HTB-17) cells were cultured in Minimum Essential Medium (MEM) Eagle's with Earle's BSS supplemented with 10\% fetal bovine serum, $2 \mathrm{mM}$ glutamine, $1 \mathrm{mM}$ sodium pyruvate, non-essential amino acid, $100 \mathrm{U} / \mathrm{mL}$ penicillin, and $100 \mu \mathrm{g} / \mathrm{mL}$ streptomycin at $37^{\circ} \mathrm{C}$ in $5 \% \mathrm{CO}_{2} 95 \%$ air. U-87MG (ATCC HTB-14) cells were cultured in the same medium but without sodium pyruvate and non-essential amino acids.

\section{Screening procedures}

U-251MG cells were plated in black 96-well plates with a clear plastic bottom (Corning Inc., Corning, NY) at a density of 20,000 cells per well. Eighty wells contained test compounds, and the first and last columns of each plate were used for vehicle control (no test compound) and negative control (cells treated with $0.5 \mathrm{U} / \mathrm{mL}$ PI-PLC (Invitrogen, Carlsbad, CA)). After overnight growth to reach confluence, cells were washed twice with PBS, then $50 \mu \mathrm{L}$ of culture medium was added to each well. Test compounds were added $(0.5 \mu \mathrm{L}$ of 2.5 $\mathrm{mM}$ DMSO stock) to each well to give $25 \mu \mathrm{M}$ final concentration and incubated with cells at $37^{\circ} \mathrm{C}$ overnight. After washing twice, cells were fixed in $4 \%$ paraformaldehyde for $15 \mathrm{~min}$ and then blocked with $1 \%$ BSA for $30 \mathrm{~min}$. Then, $50 \mu \mathrm{L}$ of a pre-mixed solution of anti-human CD55 antibody (NaM16-4D3, 1:200, Santa Cruz Biotechnology, Dallas, TX), anti-human CD59 antibody (YTH53, 1:200, Santa Cruz Biotechnology), Alexa Fluor-555 labeled goat anti-rat (A-21434, Invitrogen), and Alexa Fluor-647-labeled donkey anti-mouse IgG secondary antibody (A-31571, Invitrogen) (1:500 dilution) was then added and incubated with cells for $3 \mathrm{~h}$. After washing four times with PBS containing 0.05\% Tween-20, fluorescence was measured using a plate reader at excitation/ emission wavelengths of $555 / 565$ and $650 / 665 \mathrm{~nm}$. Relative expression was computed as follows: (compound-negative control)/(DMSO-negative control).

\section{Astrocyte cell culture}

Primary astrocyte cultures were generated from the brain cortex of neonatal rats at day 7 post-birth, as described [24] with modification. Briefly, the cerebral hemispheres were isolated and cortical tissue was minced and incubated for 15 min at $37{ }^{\circ} \mathrm{C}$ in $0.25 \%$ trypsin-EDTA. Dissociated cells were centrifuged and resuspended in Dulbecco's modified Eagle's medium (DMEM) containing 10\% FBS and 1\% penicillin/ streptomycin, and grown at $37^{\circ} \mathrm{C}$ in a $5 \% \mathrm{CO}_{2}$ incubator. After confluence (8-10 days), flasks were shaken in a rotator at $180 \mathrm{rpm}$ overnight to purify astrocytes and medium was replaced with DMEM containing 3\% FBS and $0.25 \mathrm{mM}$ dibutyryl cAMP. Cultures were maintained for an additional 2 weeks. Cultures contained $>95 \%$ astrocytes as seen by positive glial fibrillary acidic protein (GFAP) (AB5541, 1:1000, Millipore) immunofluorescence.

\section{Complement-dependent cytotoxicity (CDC)}

Astrocyte cultures were trypsinized, plated onto 96-well plates at 20,000 cells/well, and grown for $48 \mathrm{~h}$. Human complement and AQP4-IgG were added in Hank's balanced salt solution (HBSS, pH 7.2; Invitrogen), and cells were incubated at $37^{\circ} \mathrm{C}$ for $2 \mathrm{~h}$ for cytotoxicity measurement by the Alamar Blue assay (Invitrogen) as described [25]. For C3d immunostaining, astrocyte cultures were exposed to AQP4-IgG and C6-depleted serum for $2 \mathrm{~h}$, then washed and fixed with $4 \%$ paraformaldehyde (PFA). C6-depleted serum was used to visualize complement activation without cell lysis from membrane attack complex formation. After blocking, cells were incubated with anti-human C3d (1:50, Santa Cruz Biotechnology), then washed and incubated with Alexa-Fluor 555-conjugated secondary antibody for $1 \mathrm{~h}$ ( $5 \mu \mathrm{g} / \mathrm{mL}$, Invitrogen). 


\section{Intracerebral injection model}

Mice were housed and bred in the UCSF Laboratory Animal Resource Center. AQP4-IgG (7.5 $\mu \mathrm{g})$ was delivered by intracerebral injection as described $[13,26]$ with modification. Adult wild-type male mice (age 8-10 weeks; weight $25-30 \mathrm{~g}$ ) in a CD1 genetic background were anesthetized with ketamine $(100 \mathrm{mg} / \mathrm{kg})$ and xylazine $(10 \mathrm{mg} / \mathrm{kg})$ and mounted on a stereotaxic frame. A midline scalp incision was made, and a burr hole of 1-mm diameter was drilled on each side of the skull 0.5 $\mathrm{mm}$ anterior and $2 \mathrm{~mm}$ lateral to the bregma. A glass pipette with $40-\mu \mathrm{m}$ diameter tip was inserted at a depth of $3 \mathrm{~mm}$ to infuse $7.5 \mu \mathrm{g}$ AQP4-IgG (or control IgG) and $1 \mu \mathrm{L}$ human complement in a total volume of $3 \mu \mathrm{L}$ over $10 \mathrm{~min}$ by pressure injection. After injection, the glass pipette was kept in place for $10 \mathrm{~min}$ before slow withdrawal over 5 min to prevent leaking. At day 6, mice were deeply anesthetized and transcardially perfused with $200 \mathrm{~mL}$ heparinized PBS and $200 \mathrm{~mL}$ of $4 \%$ PFA in PBS. Brains were removed and post-fixed for $4 \mathrm{~h}$ in $4 \%$ PFA and cryoprotected in $20 \%$ sucrose. Serial frozen coronal sections (thickness $7 \mu \mathrm{m}$ ) were cut on a cryostat (Leica CM 1900, Leica Biosystem, Inc., Buffalo Grove, IL).

\section{Immunofluorescence}

U-251MG and primary astrocyte cultures were grown on glass coverslips for $24 \mathrm{~h}$, then incubated with test compound for another $24 \mathrm{~h}$. Cells were then fixed in $4 \%$ PFA for 15 min. After blocking with $1 \%$ BSA in PBS, cells were incubated with primary antibody: anti-human CD55 (NaM16-4D3, 1:100), anti-human CD59 (YTH53, 1:700), anti-rat CD55 (RDIII-7 1:50, Hycult Biotech, Plymouth Meeting, PA), anti-rat CD59 (clone TH9, 1:50, LifeSpan Biosciences, Seattle, WA), or anti-human C3d (003-05, 1:50, Santa Cruz Biotechnology) for $1 \mathrm{~h}$ at room temperature. Cells were then washed with PBS and incubated with the appropriate species-specific Alexa Fluor-conjugated secondary antibody for $1 \mathrm{~h}$ (Alexa Fluor-488-labeled goat anti-rat (A-11006) and Alexa Fluor-555 labeled goat anti-mouse IgG secondary antibody (A-21424), $5 \mu \mathrm{g} / \mathrm{mL}$ each, Invitrogen). Slides were washed four times with PBS and mounted using Prolong $^{\text {Tw }}$ Gold antifade reagent with DAPI (Invitrogen) counterstain for visualization of immunofluorescence on a Leica fluorescence microscope or Nikon confocal microscope.

Frozen sections of mouse brain, spinal cord, optic nerve, and skeletal muscle were post-fixed in 4\% PFA for $5 \mathrm{~min}$ and incubated in blocking solution as described [18, 27]. Slides were then incubated for $2 \mathrm{~h}$ with antibodies against CD55 (NaM16-4D3, 1:50), CD59 (7A6, $2 \mu \mathrm{g} / \mathrm{mL}$, Hycult Biotech), AQP4 (H-80, 1:200, Santa Cruz Biotechnology), C3d (003-05, 1:100, Santa Cruz Biotechnology), C5b-9 (aE11, 1:100, Santa Cruz Biotechnology), myelin basic protein (MBP, C-16, 1:100, Santa Cruz Biotechnology), ionized calcium-binding adaptor molecule 1 (Iba-1, 019-19741, 1:1000, Wako, Richmond, VA), or endothelial cell marker (isolectin B4-FITC conjugated, ALX-650-001F-MC05, $10 \mu \mathrm{g} / \mathrm{mL}$, Enzo Life Sciences, Farmingdale, NY), followed by the appropriate secondary antibody for $1 \mathrm{~h}$ (Alexa Fluor-488 labeled donkey anti-rabbit (A-21206), Alexa Fluor-488-labeled donkey anti-goat (A-11055), Alexa Fluor-555-labeled donkey anti-mouse (A-31570), and Alexa Fluor-555-labeled donkey anti-rabbit IgG secondary antibody (A-31572), $5 \mu \mathrm{g} / \mathrm{mL}$ each, Invitrogen).

\section{Image analysis}

Fluorescence signal was quantified using Image $(\mathrm{NIH})$. For measurements in cell cultures, mean pixel fluorescence intensity was measured using full-field images, with subtraction of background as determined using three or more areas devoid of cells. Relative fluorescence signal was reported as the ratio of drug-treated cells to DMSO-treated cells. Full-field images were also analyzed for measurements of CD55 immunofluorescence in tissues, in which background was determined after thresholding to exclude $\sim 25 \%$ of the brightest pixels; robustness of the analysis was verified in an independent analysis that used thresholding to identify regions of interest around cell structures (not reported). For mouse brain, quantification of lesion area was done by image analysis as described [17] in which AQP4 and MBP immunonegative areas, and C5b-9 immunopositive areas, were defined by hand and areas quantified using ImageJ.

\section{RT-PCR}

cDNA from reverse-transcribed mRNA isolated from U-251MG cells was PCR amplified with the following primers: 5'-TGACTGTGGCCTTCCCCCAGAT-3' and 5'-GTGTTACATGAGAAGGAGATGG-3', 168-640 (CD 55), and 5'-TGACGGGGTCACCCACACTGTGCCCAT CTA-3' and 5'-CTAGAAGCATTGCGGTGGACGATG GAG GG-3', 478-1128 ( $\beta$-actin) [28] in an Applied Biosystems 2720 Thermal Cycler (Applied Biosystems, Foster City, CA). PCR conditions were at $94^{\circ} \mathrm{C}$ for $5 \mathrm{~min}$ followed by 30 cycles of $94{ }^{\circ} \mathrm{C}$ for $30 \mathrm{~s}$ and $54^{\circ} \mathrm{C}$ for $1 \mathrm{~min}$, with final extension at $72^{\circ} \mathrm{C}$ for $7 \mathrm{~min}$. After amplification, the PCR products were run on a $2 \%$ agarose gel with ethidium bromide and photographed by Axygen $\mathrm{Gel}^{\circ}$ Documentation System: Model GDBL-1000 (Fisher Scientific, Waltham, MA). The images were quantified using Image); relative mRNA expression was calculated by the ratio of target gene expression to reference gene ( $\beta$-actin).

\section{Statistics}

Data are presented as mean \pm SEM. Statistical analysis was performed using Prism 5 GraphPad Software 
package (San Diego, CA). Normal distribution of all data was confirmed by the Shapiro-Wilk test. Statistical comparisons were made using unpaired Student's $t$ test for comparisons between two groups. Differences were considered significant at $P<0.05\left(*=P \leq 0.05,{ }^{* * *}=P \leq 0.01\right)$.

\section{Results}

\section{Complement regulator screen}

An assay suitable for high-throughput screening was established to identify compounds that upon many hours of incubation could increase the expression of CD55 or CD59 in astrocytes. Because compounds were sought that acted by a transcriptional mechanism, a human astrocyte cell line was chosen with low or moderate endogenous expression of CD55 and CD59 in order to produce measurable signals in the screening assay and in which baseline expression is sufficiently low to permit upregulation. The immortalized human astrocyte cell lines U-87MG, U-251MG, and U-373MG were tested. CD55 and CD59 immunofluorescence showed different levels of expression in the three cell lines (Fig. 1). Little fluorescence was seen in control cells following treatment with PI-PLC, which cleaves the extracellular antigen-containing portions of CD55 and CD59. U-373MG cells were not used for screening because of their very low CD55 immunofluorescence. U-87MG cells were not used for screening because they detached easily during washing steps and because of their high baseline CD55 expression that is likely to be near or at the maximum level. U-251MG cells were selected for screening based on their moderate expression of CD55 and CD59, which provided a measurable signal with sufficient room for upregulation.

Figure 2a diagrams the cell-based screening assay in which suitable antibodies were chosen for two-color detection of CD55 and CD59 expression in the same cells without cross-talk. The assay used a mouse anti-human primary CD55 antibody and rat anti-human primary CD59 antibody, with appropriate fluorescent secondary antibodies. Examples of assay data are shown in Fig. 2b. PI-PLC strongly reduced fluorescence for both CD55 and CD59 detection, demonstrating assay specificity; $Z$-factor analysis showed assay reliability ( $Z^{\prime}$-factors 0.52 and 0.48 , respectively). The assay did not include "positive controls" because there are no known compounds that upregulate CD55 or CD59 expression in astrocytes.

Screening was done on collections of approved and investigational drugs, and nutraceuticals, recognizing the benefits of drug repurposing for human clinical testing. The screen, which was done at a drug/nutraceutical concentration of $25 \mu \mathrm{M}$, did not produce compounds that significantly increased CD59 expression (cut-off 1.5-fold); however, compounds that increased CD55 expression by $>2$-fold were identified, including several statins (atorvastatin, simvastatin, lovastatin, mevastatin) and phorbal 12-myristate 13-acetate. Because of their generally good brain penetration and established safety profile, further studies were done on statins. Figure 2c shows chemical structures of FDA-approved statins that were investigated, each of which contains a dihydroxyheptanoic acid component that is structurally similar to the 3-hydroxy-3-methyl-glutaryl-coenzyme A (HMG CoA) substrate in the cholesterol biosynthetic pathway.

\section{CD55 upregulation by statins}

U-251MG cells were incubated with each of the statins at $25 \mu \mathrm{M}$ for $24 \mathrm{~h}$. Figure 3a shows representative CD55 and CD59 immunofluorescence, with the fold-increased expression summarized in Fig. 3b. Most statins substantially increased CD55 expression, though none of the compounds increased CD59 expression. The broad activity of statins on CD55 expression suggests action on their well-known target, HMG-CoA reductase, rather than off-target effects. The lack of effect of pravastatin may be related to its high hydrophilicity and consequent low cell permeability. Atorvastatin, which is the seventh most commonly prescribed drug in the US, was selected for further studies because of its penetration into the

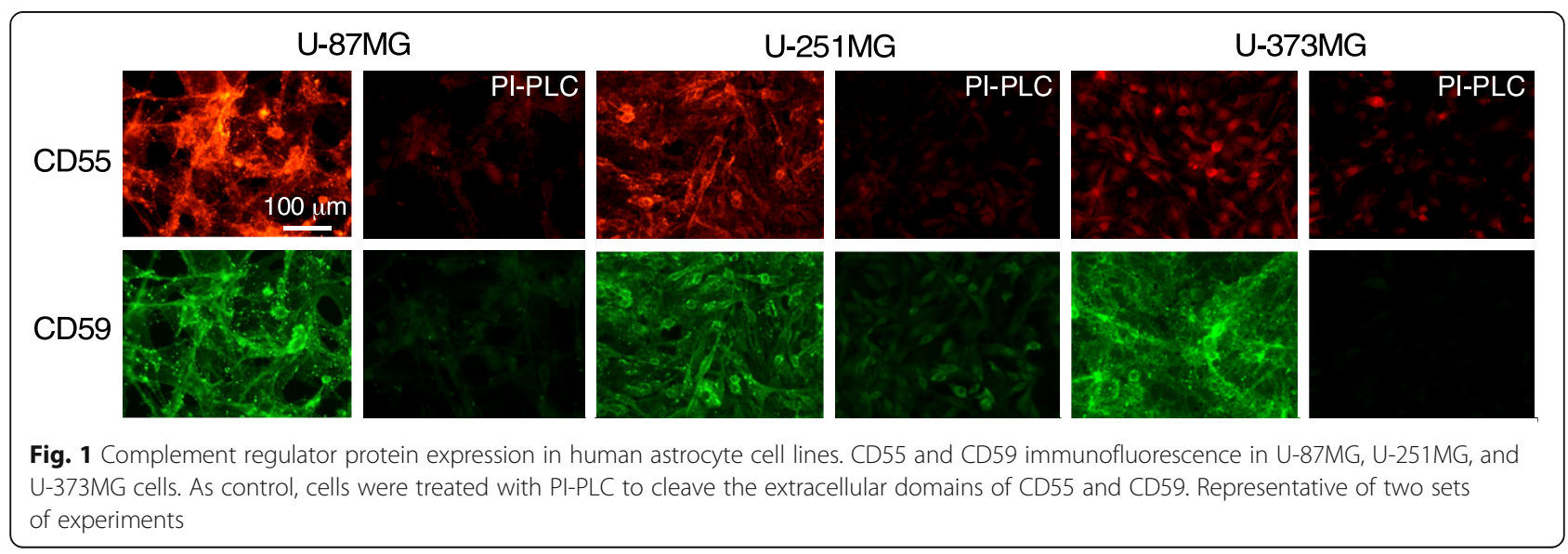




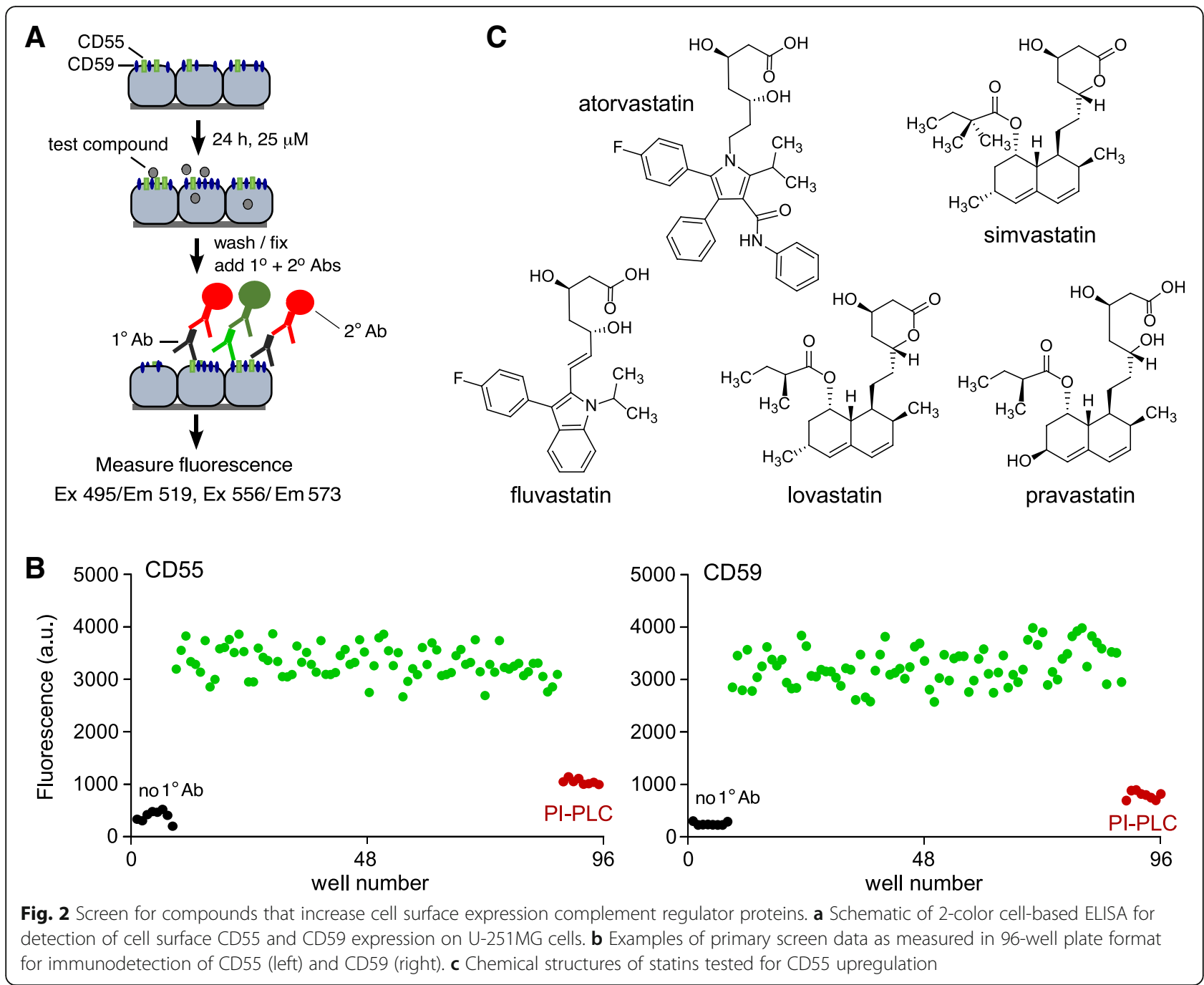

central nervous system [29, 30], as well as its favorable immunosuppressive and immunomodulatory actions [31, 32], which are of potential therapeutic benefit in NMO.

Atorvastatin concentration-dependence data for CD55 upregulation in U-251MG cell cultures showed an $\mathrm{EC}_{50}$ of 1$2 \mu \mathrm{M}$ at $24-\mathrm{h}$ incubation time (Fig. 4a). A time course study at $5 \mu \mathrm{M}$ atorvastatin showed slow onset of action, with a $50 \%$ increase in CD55 expression at 8-16 h, which is consistent with a transcriptional upregulation mechanism (Fig. 4b). In support of a transcriptional mechanism, RT-PCR showed significant increased CD55 transcript in U-251MG cells treated for $48 \mathrm{~h}$ with $1 \mu \mathrm{M}$ atorvastatin, with comparable $\beta$-actin transcript (Fig. 4c) (fold increase comparing with vs. without atorvastatin, $1.6 \pm 0.1$, SEM, $n=3, P<0.01$ ). Cycloheximide block of protein synthesis largely prevented the increased CD55 expression by atorvastatin (Fig. 4d).

\section{Mechanism of CD55 upregulation by atorvastatin}

Statins inhibit cholesterol biosynthesis as well as the formation of isoprenoid intermediates farnesyl pyrophosphate (farnesyl-PP) and geranylgeranyl pyrophosphate (geranylgeranyl-PP), which affects posttranslational modification of a variety of signaling proteins, including small GTPase RhoA, Cdc42, Rac1, Rab, and heterotrimeric G proteins (Fig. 5a). Selective modulators of components of the acetyl-CoA signaling pathway were tested for their effects on CD55 expression. As shown in Fig. 5b and c, inclusion of mevalonic acid (MVA) with atorvastatin for $24 \mathrm{~h}$ prevented the atorvastatin effect, supporting the conclusion that atorvastatin upregulation of CD55 involves inhibition of HMG-CoA reductase, as mevalonate bypasses the enzyme block. The squalene synthase inhibitor zaragozic acid did not increase CD55 expression, indicating that the atorvastatin effect is not related to its cholesterol-lowering action. Geranylgeraniol (GGOH), an alcohol precursor of geranylgeranyl pyrophosphate (geranylgeranyl-PP), largely blocked the atorvastatin-induced CD55 upregulation, suggesting that action of atorvastatin on the geranylgeranyl transferase pathway is responsible 


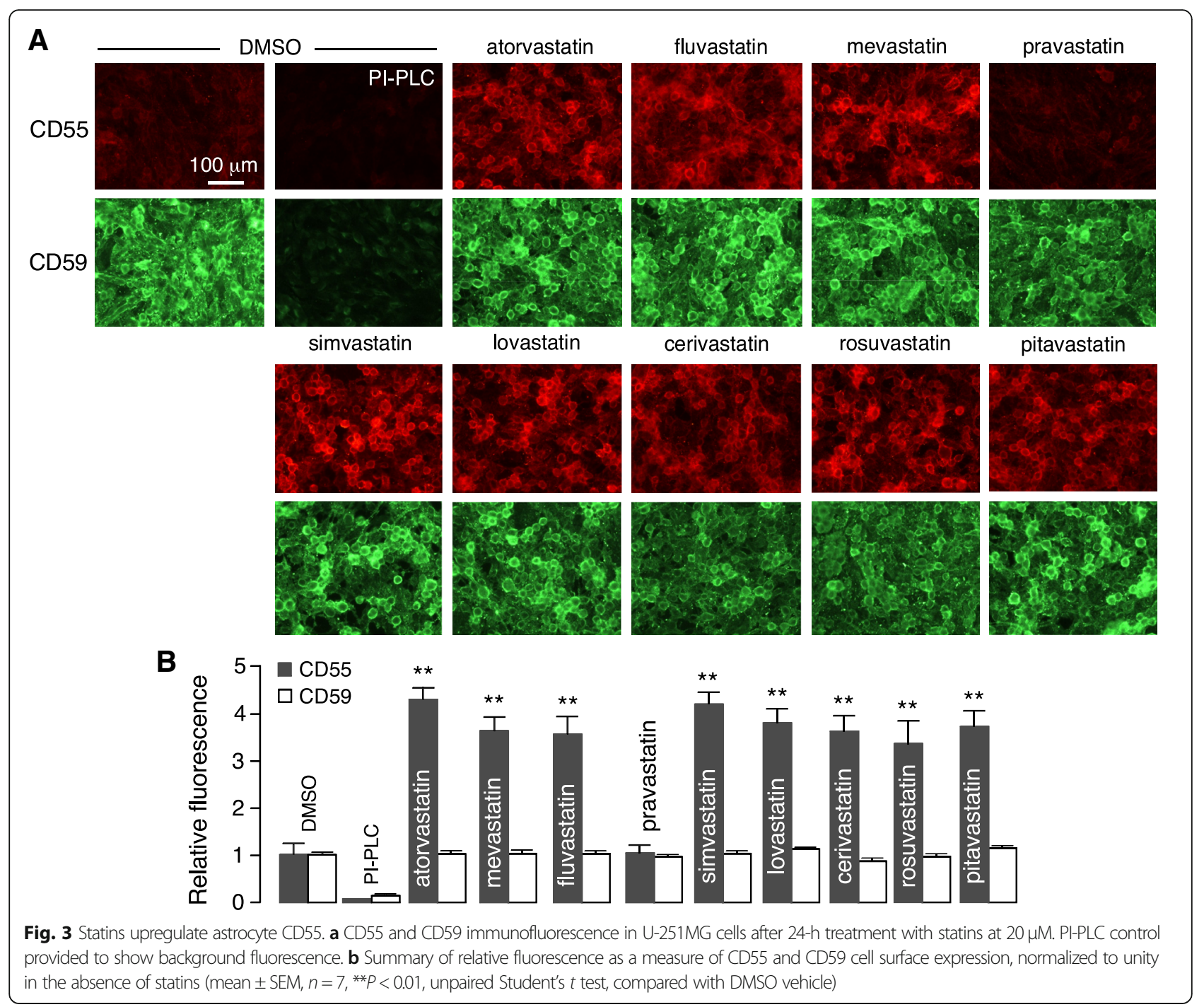

for CD55 upregulation. In support of this conclusion, the geranylgeranyl transferase inhibitor GGTI-286 (in the absence of atorvastatin) recapitulated the CD55 upregulation seen with atorvastatin. The RhoA inhibitor fasudil did not increase CD55 expression.

\section{Atorvastatin increases CD55 expression in astrocyte cultures and mouse brain}

Atorvastatin upregulation of CD55 was investigated in primary astrocyte cultures and in mice in vivo. Primary astrocyte cultures were generated from neonatal rat brains and differentiated by inclusion of dibutyryl cAMP in the culture medium. Atorvastatin for $24 \mathrm{~h}$ increased CD55 expression by up to $\sim 3$-fold, without significant effect on CD59 expression (Fig. 6a). CD55 immunofluorescence showed a somewhat heterogeneous response from cell to cell, both in CD55 expression at baseline and after atorvastatin treatment. To determine whether CD55 upregulation in rat astrocyte cultures was protective against complement-dependent cytotoxicity, cytotoxicity was measured in control cells and cells incubated for $24 \mathrm{~h}$ with atorvastatin prior to addition of AQP4-IgG and human complement. Atorvastatin significantly reduced cytotoxicity in a concentration-dependent manner (Fig. 6b). C3d immunofluorescence of atorvastatin-treated astrocyte cultures exposed to AQP4-IgG and C6-depleted complement was greatly reduced (Fig. 6b, inset), consistent with the cytoprotective action of atorvastatin.

For experiments in vivo, mice were orally administered atorvastatin at $10 \mathrm{mg} / \mathrm{kg}$ daily for 3 days, a dose regimen similar to that used in prior in vivo brain studies in mice [33-35]. CD55 immunofluorescence showed increased CD55 immunofluorescence in the brain, optic nerve, and spinal cord of atorvastatin-treated mice, though no significant effect in skeletal muscle (Fig. 6c-e). CD59 expression was similar in control and atorvastatin-treated mouse brain (Fig. 6c, left). The pattern of CD55 expression in atorvastatin-treated mouse brain was similar to 


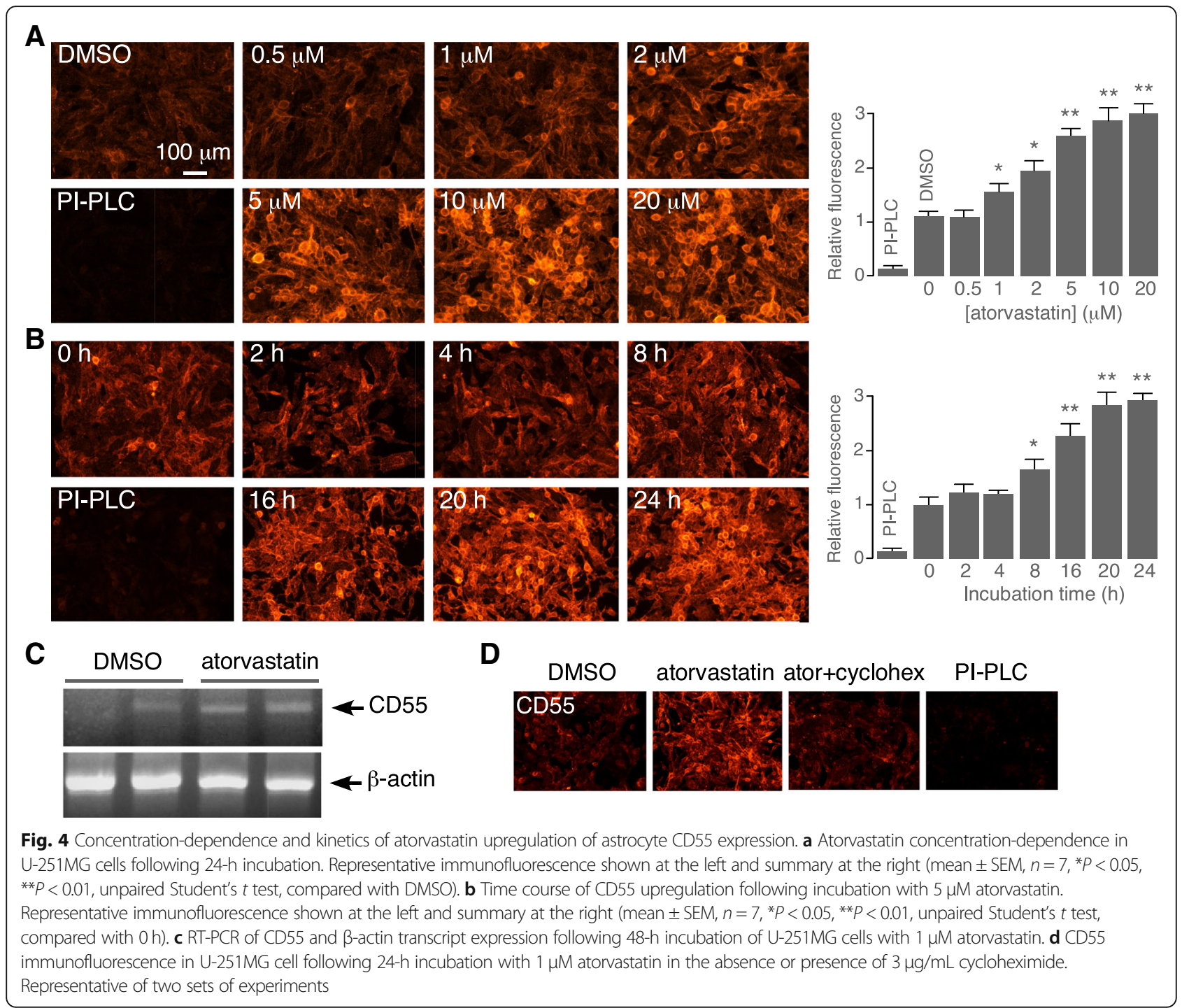

that of AQP4, the astrocyte target of NMO autoantibody (Fig. 6c, right) and that of an endothelia cell marker (FITC-Isolectin B4) (Fig. 6f); however, by light microscopy the relative increase in CD55 expression in astrocytes versus endothelia and other brain cells cannot be resolved. The expression of AQP4 in central nervous system tissues and skeletal muscle was similar in the control and atorvastatin-treated mice (Fig. 6c-e).

\section{Atorvastatin reduces pathology in an experimental mouse model of NMO}

Atorvastatin efficacy in reducing NMO pathology was tested in an established experimental animal model of NMO. As diagrammed in Fig. 7a, mice received atorvastatin at $20 \mathrm{mg} / \mathrm{kg}$ daily for 3 days prior to intracerebral injection of AQP4-IgG and human complement, with control mice receiving saline instead of atorvastatin [13, $26,36]$. Atorvastatin was continued daily until sacrifice on day 6 at which time brains were processed for immunofluorescence. Figure $7 \mathrm{~b}$ shows reduced loss of $\mathrm{AQP} 4$ and MBP, and reduced Iba-1 immunofluorescence, in the brain of atorvastatin-treated mice in a region surrounding the AQP4-IgG injection site compared with vehicle-treated mice. These findings demonstrate reduced astrocytopathy (AQP4), myelin loss (MBP), and inflammation (Iba-1) with atorvastatin treatment. Lesion size, as quantified by the areas of AQP4 and MBP loss, was significantly reduced in atorvastatin-treated mice (Fig. 7d). There was also an increase in immunofluorescence of markers of complement activation (C3d, C5b-9) (Fig. 7c, d) and inflammation (Iba-1) (Fig. 7d).

\section{Discussion}

The purpose of this study was to identify a drug or nutraceutical that increases the expression of complement regulator protein(s) in astrocytes, for possible repurposing in 


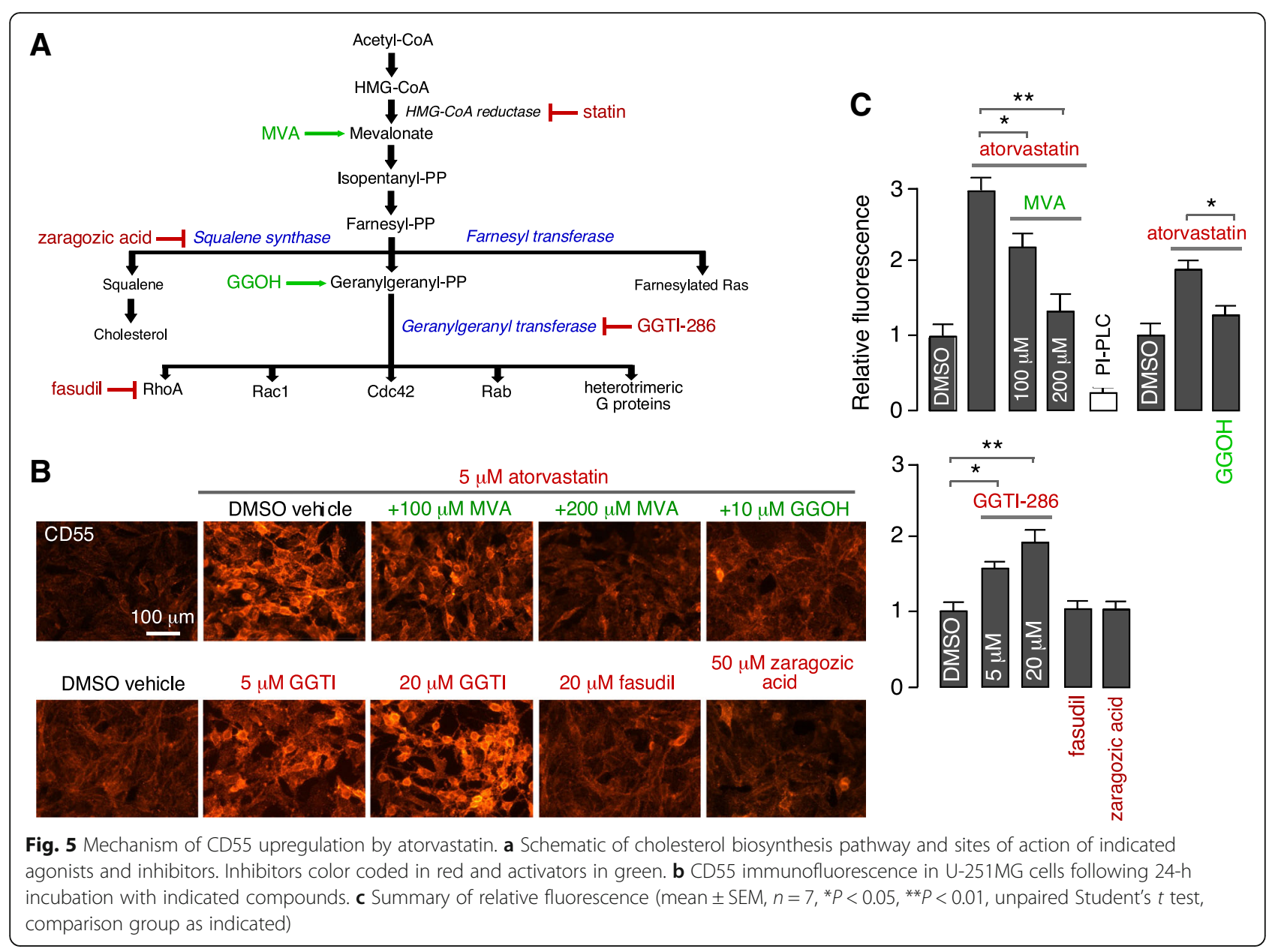

NMO. The motivation for this work is the central importance of complement-mediated cytotoxicity in AQP4-IgG seropositive NMO, and the predicted importance, as demonstrated in experimental animal models $[15,17,18]$ and by theoretical considerations [37], of complement regulator proteins in modulating complement action on target cells. Though there has been considerable research on complement mechanisms in NMO pathogenesis and on the development and testing of complement-targeted therapeutics $[16,25,38]$, there has been relatively little work on complement regulator proteins in NMO. Increasing the expression or activity of complement regulator proteins on target cells is a logical extension of complement inhibitor therapy for NMO, with the potential advantage of avoiding the immunosuppressive actions of global complement inhibition and their concomitant side effects. We found evidence here for atorvastatin as a transcriptional upregulator of CD55 in astrocytes by a mechanism that involves the geranylgeranyl transferase pathway. The demonstrated consequences of CD55 upregulation included inhibition of complement-dependent cytotoxicity in astrocyte cultures in vitro and reduced pathology in an experimental mouse model of NMO in vivo.
Various physiological factors and drugs have been reported to increase expression of complement regulator proteins in human endothelial cells in the umbilical vein, skin, and aorta, which was proposed to have relevance to inflammatory diseases involving complement, including atherosclerosis and rheumatoid arthritis [39-43]. Physiological factors, including TNF- $\alpha$, interferon- $\gamma$, and basic fibroblast growth factor (bFGF), were reported to increase CD55 expression by a protein kinase $\mathrm{C}$ (PKC)-independent pathway, whereas the vascular endothelial growth factor (VEGF) was reported as PKC-dependent [39, 40, 42]. Drugs including atorvastatin and simvastatin have been reported to increase complement regulator protein expression in endothelia $(41,43)$, which was proposed to explain the anti-inflammatory effect of statins on the cardiovascular system apart from their cholesterol-lowering action [44, 45].

Statins have pleotropic biological actions, several of which are of a potential benefit in NMO. Statin inhibition of HMG-CoA reductase blocks cholesterol biosynthesis, its well-described action as a lipid-lowering drug. Statins also inhibit biosynthesis of the isoprenoid 


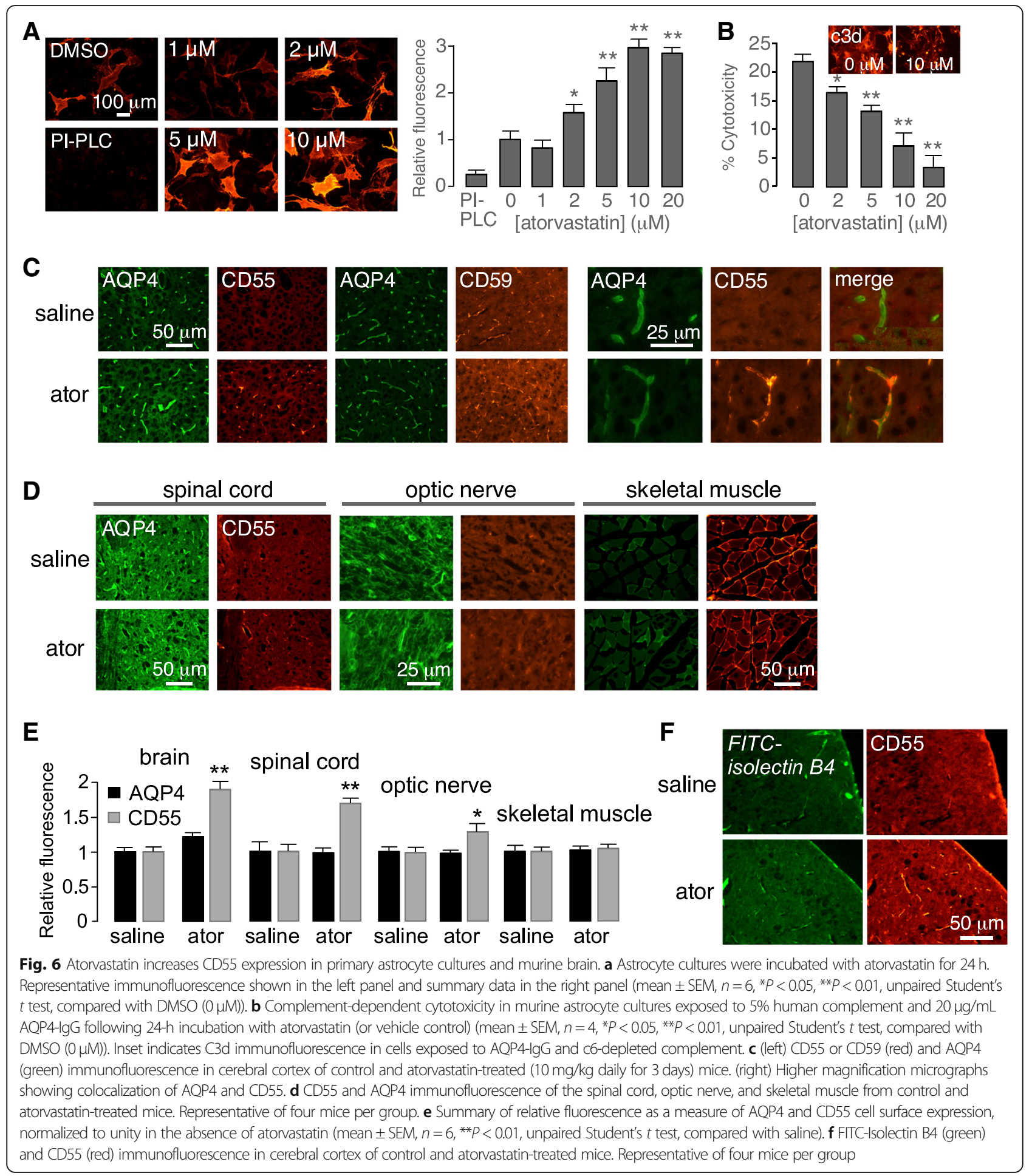

intermediates farnesyl pyrophosphate and geranylgeranyl pyrophosphate [31, 44]. Statin-induced upregulation of CD55 in endothelial cells, and consequent protection against complement-mediated injury, was reported to involve inhibition of geranylgeranyl transferase (GGTase) and RhoA [41, 46]. The mechanistic studies here in Fig. 5b suggest that CD55 upregulation by atorvastatin in astrocytes also involves GGTase inhibition. Atorvastatin has also been reported to cause CD59 upregulation in hypoxia in a model of rheumatoid arthritis by a mechanism involving nitric oxide-dependent GGTase inhibition [43]. 


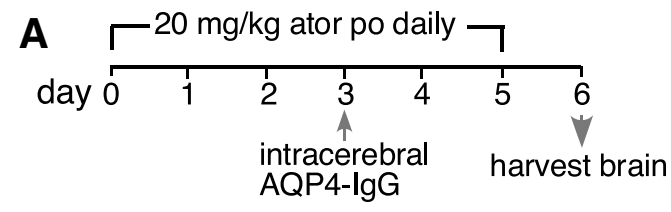

B
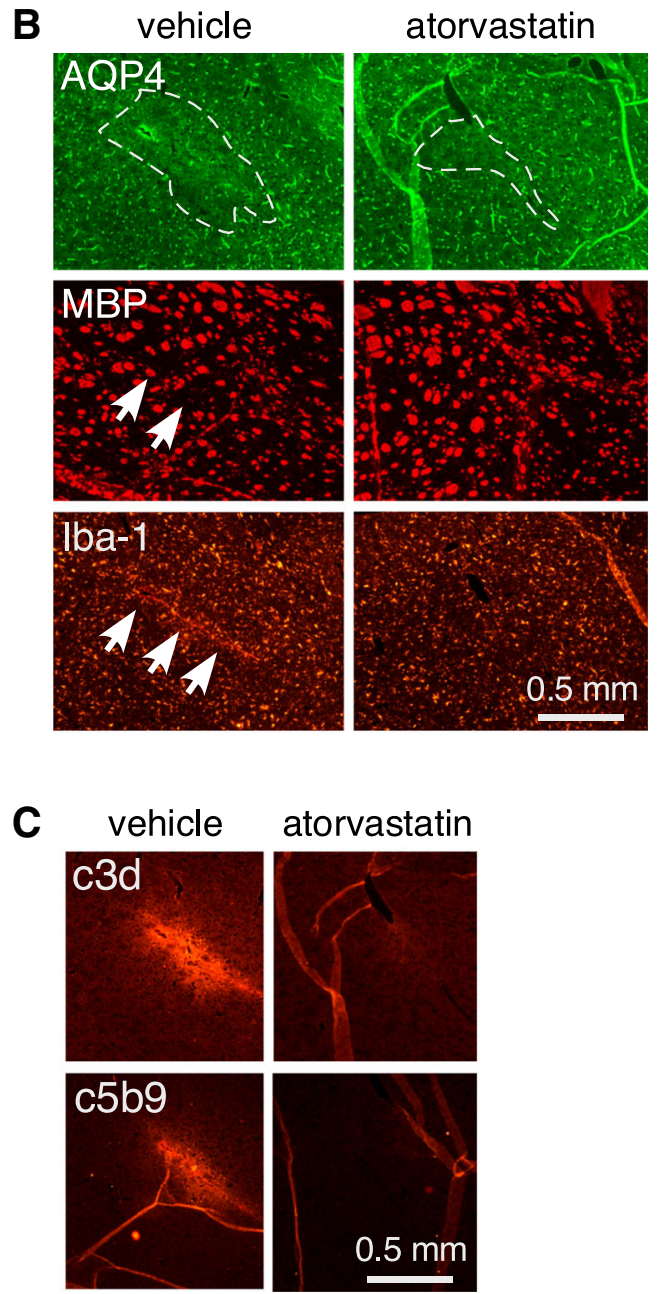

D
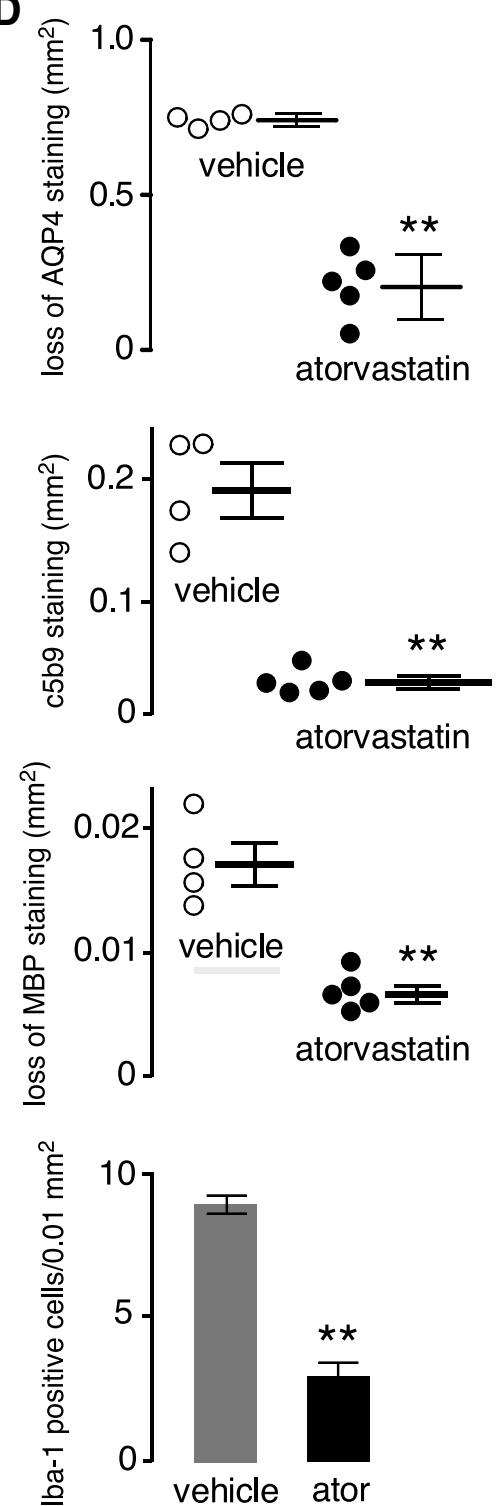

Fig. 7 Atorvastatin reduces NMO pathology in an experimental animal model of NMO produced by passive transfer of AQP4-lgG. a Experimental protocol. Mice received atorvastatin $20 \mathrm{mg} / \mathrm{kg} /$ day for 3 days, or saline vehicle, prior to intracerebral injection of $7.5 \mu \mathrm{g} \mathrm{AQP4-lgG}$ and $1 \mu \mathrm{L}$ human complement. Mice were sacrificed on day 6. $\mathbf{b}$ Immunofluorescence of AQP4, MBP, and Iba-1 in the brain at day 6 showing AQP4-lgG-injected hemisphere from control and atorvastatin-treated mice. Representative of experiments on 4-5 mice per group. c Immunofluorescence of activated complement proteins C3d and C5b-9 at day 6 from control and atonvastatin-treated mice. Representative of staining done on 4-5 mice per group. $\mathbf{d}$ Summary of lesion areas (AQP4, c5b-9, MBP, in $\mathrm{mm}^{2}$ ) and Iba-1 cell positivity (positive cells per $0.01 \mathrm{~mm}^{2}$ ), showing data from individual mice (mean \pm SEM, $n=4-5,{ }^{* *} P<0.01$, unpaired Student's $t$ test, compared with vehicle)

Several GGTase inhibitors (GGTIs) are in development as potential anticancer drugs [47-49]. GGTI-286, a potent and selective GGTase inhibitor, is in preclinical development, and GGTI-2418 is in a Phase I clinical trial for treatment of breast cancer and multiple myeloma [47-52]. Our data here support selective inhibition of GGTase as the mechanism of stain-induced CD55 upregulation in astrocytes. GGTIs may thus provide an alternative approach to increase CD55 expression without altering cholesterol biosynthesis. We note, however, that although a large body of literature supports the safety of statins, the safety of drugs that target other steps of the cholesterol biosynthesis pathway must be demonstrated. In addition, there are theoretical adverse effects of increasing complement regulator expression such as impairment of immune surveillance in cancer.

The ability of drugs to cross the blood-brain barrier is an important consideration in NMO therapy. The 
penetration of statins into the central nervous systems varies with their structure and lipophilicity. Lipophilic statins (atorvastatin, lovastatin, fluvastatin, pitavastatin, simvastatin) can penetrate the blood-brain barrier passively, while the hydrophilic statin pravastatin does not $[29,30]$. Among the lipophilic statins, lovastatin has been reported in rat studies to best cross the blood-brain barrier [29]. However, lovastatin and simvastatin are inactive lactone prodrugs that require in vivo hydrolysis to give the pharmacologically active form. Atorvastatin is an active drug with high cellular and blood-brain barrier penetration. Atorvastatin has been reported to have neuroprotective effects in animal models of brain pathology associated with neuroinflammation, including traumatic brain injury and experimental autoimmune encephalomyelitis [33, 35]. Atorvastatin has also been reported to have immunomodulatory actions involving alteration of $\mathrm{T}$ and antigen-presenting cell (APC) function, and inhibition of immune cell invasion [31-33]. These immunomodulatory effects of atorvastatin may be beneficial in $\mathrm{NMO}$, apart from its action on complement regulator expression.

An atorvastatin dose of $10-20 \mathrm{mg} / \mathrm{kg} /$ day orally was chosen here for in vivo mouse experiments. This dose was based on pharmacokinetic data and prior studies of neuroprotection in rodents in which $10-50 \mathrm{mg} / \mathrm{kg}$ atorvastatin was needed to achieve similar plasma concentrations as in humans [30, 33-35, 53-55], in which the maximum recommended daily dose is $80 \mathrm{mg} /$ day, or $1-2 \mathrm{mg} / \mathrm{kg}$. Atorvastatin may thus confer neuroprotection in humans at the typically used human doses.

Questions in targeting complement regulator proteins for NMO therapy include which complement regulator proteins to target and on what cell types and the magnitude of upregulation needed to confer clinical benefit. There are available data regarding CD59 in NMO from experimental animal models. Transgenic knockout of CD59 greatly increases the susceptibility of mice and rats to development of NMO pathology following passive transfer of AQP4-IgG into central nervous system tissues $[15,17,18]$. CD59 upregulation by lentivirus is associated with reduced NMO pathology [21]. Mathematical modeling showed that theoretical increases in CD59 expression could prevent cell swelling induced by AQP4-IgG and human complement [37]. Upregulation of CD55, but not of CD59, would inhibit the generation of complement anaphylotoxins (C3a, C4a, and $\mathrm{C} 5 \mathrm{a})$, as CD55 inhibits the assembly and accelerates the decay of C3- and C5-convertase. Additional potential advantages of upregulation of CD55 vs. CD59 for NMO therapy include the greater-fold increase in CD55 expression possible because of its low baseline expression compared to CD59 and inhibition of a different step in the complement pathway and hence possible synergy with CD59.
Another theoretical advantage is the ostensible difference in the subcellular distribution of CD55 vs. CD59, as CD59 has been reported to be expressed at relatively lower levels in astrocyte end-feet where AQP4 is concentrated [56].

Though the data herein provide proof of concept for the potential therapeutic utility of CD55 upregulation by atorvastatin in seropositive $\mathrm{NMO}$, several limitations are noted. The demonstration of CD55 upregulation by atorvastatin in astrocyte cultures and mouse brain does not ensure that clinically significant CD55 upregulation will occur in NMO-affected tissues in humans at tolerated doses of atorvastatin. As atorvastatin inhibits cholesterol biosynthesis, the possibility exists, at least theoretically, for inhibition of remyelination $[57,58]$, which might account for the inconclusive benefit of statin treatment in multiple sclerosis [59]. Finally, upregulation of CD55 alone does not target other effectors of NMO pathogenesis, such as AQP4-IgG production and levels, antibody-dependent cellular cytotoxicity, the peripheral complement system, and $\mathrm{T}$ cell responses, though atorvastatin could be combined with other currently used or investigational NMO therapeutics such as immunosuppressants, plasma exchange, complement inhibitors, and cell-targeted drugs.

\section{Conclusions}

In conclusion, pharmacological upregulation of complement regulator proteins such as CD55 represents a novel approach for therapy of AQP4-IgG seropositive NMO. Since upregulation of complement regulator proteins does not interfere with the classical, alternative, or lectin complement pathways, the side-effect profile might be relatively minimal. Upregulation of CD55 expression by atorvastatin is predicted to not only inhibit formation of the complement membrane attack complex on astrocytes and nearby bystander cells, but also inhibit the formation of complement anaphylotoxins that promote inflammation and cellular injury. Clinical evaluation of atorvastatin in seropositive NMO therefore seems warranted based on its established safety profile and multiple potential beneficial actions. Retrospective analysis of NMO patients treated with statins for other indications may be informative as well.

\footnotetext{
Abbreviations

AQP4: Aquaporin-4; AQP4-lgG: Aquaporin-4-immunoglobulin G;

CDC: Complement-dependent cytotoxicity; DMEM: Dulbecco's modified Eagle

medium; farnesyl-PP: Farnesyl pyrophosphate; FBS: Fetal bovine serum;

geranylgeranyl-PP: Geranylgeranyl pyrophosphate; GFAP: Glial fibrillary acidic

protein; GGTase: Geranylgeranyl transferase; GGTI: Geranylgeranyl transferase inhibitor; HBSS: Hank's balanced salt solution; HMG-CoA: 3-Hydroxy-3-methylglutaryl-coenzyme A; MBP: Myelin basic protein; MVA: Mevalonic acid; NMO: Neuromyelitis optica; PFA: Paraformaldehyde; PI-PLC: Phosphatidylinositolspecific phospholipase C; PKC: Protein kinase C
} 


\section{Acknowledgements}

We thank Dr. Jeffrey Bennett (Univ. Colorado Denver, Aurora, CO) for providing recombinant monoclonal NMO antibody.

\section{Funding}

This work was supported by the Guthy-Jackson Charitable Foundation, and grants EY13574, EB00415, DK35124 and DK72517 from the National Institutes of Health.

\section{Availability of data and materials}

All data supporting the conclusions of this manuscript are provided in the text and figures.

\section{Authors' contributions}

$L T$ and $T J$ performed the in vitro and in vivo experiments and analyzed the data. LT and ASV designed the experiments. LT, TJ, MRY, and ASV wrote and edited the manuscript. ASV and MRY conceived the original idea for this study. All authors read and approved the final manuscript.

\section{Ethics approval and consent to participate}

Procedures were approved by the UCSF Institutional Animal Care Use Committee (IACUC). Consent to participate is not applicable.

\section{Consent for publication}

Not applicable.

\section{Competing interests}

The authors declare that they have no competing interests.

\section{Publisher's Note}

Springer Nature remains neutral with regard to jurisdictional claims in published maps and institutional affiliations.

\section{Author details}

'Departments of Medicine and Physiology, University of California, 1246 Health Sciences East Tower, 513 Parnassus Ave, San Francisco, CA 94143-0521, USA. ${ }^{2}$ Department of Neurology, Second Xiangya Hospital of Central South University, Changsha 410011, Hunan, People's Republic of China. ${ }^{3}$ Department of Medicine, David Geffen School of Medicine, University of California, Los Angeles, CA 90024, USA. " Division of Molecular Medicine, Harbor-UCLA Medical Center, Torrance, CA 90502, USA.

\section{Received: 4 January 2019 Accepted: 26 February 2019}

\section{Published online: 09 March 2019}

\section{References}

1. Lennon VA, Kryzer TJ, Pittock SJ, Verkman AS, Hinson SR. IgG marker of optic-spinal multiple sclerosis binds to the aquaporin-4 water channel. J Exp Med. 2005;202(4):473-7.

2. Misu T, et al. Loss of aquaporin 4 in lesions of neuromyelitis optica: distinction from multiple sclerosis. Brain. 2007;130(Pt 5):1224-34.

3. Jarius S, Wildemann B. AQP4 antibodies in neuromyelitis optica: diagnostic and pathogenetic relevance. Nat Rev Neurol. 2010;6(7):383-92.

4. Papadopoulos MC, Verkman AS. Aquaporin 4 and neuromyelitis optica. Lancet Neurol. 2012;11(6):535-44.

5. Hoftberger $\mathrm{R}$, Lassmann $\mathrm{H}$. Inflammatory demyelinating diseases of the central nervous system. Handb Clin Neurol. 2017;145:263-83.

6. Greenberg BM, et al. Rituximab dosing and monitoring strategies in neuromyelitis optica patients: creating strategies for therapeutic success. Mult Scler. 2012;18(7):1022-6.

7. Papadopoulos MC, Bennett IL, Verkman AS. Treatment of neuromyelitis optica: state-of-the-art and emerging therapies. Nat Rev Neurol. 2014;10(9):493-506.

8. Kitley J, Palace J. Therapeutic options in neuromyelitis optica spectrum disorders. Expert Rev Neurother. 2016;16(3):319-29.

9. Bruscolini A, et al. Diagnosis and management of neuromyelitis optica spectrum disorder. Autoimmun Rev. 2018;17(3):195-200.

10. Lucchinetti $C F$, et al. A role for humoral mechanisms in the pathogenesis of Devic's neuromyelitis optica. Brain. 2002;125(Pt 7):1450-61.

11. Hengstman GJ, Wesseling P, Frenken CW, Jongen PJ. Neuromyelitis optica with clinical and histopathological involvement of the brain. Mult Scler. 2007;13(5):679-82.
12. Roemer SF, et al. Pattern-specific loss of aquaporin-4 immunoreactivity distinguishes neuromyelitis optica from multiple sclerosis. Brain. 2007; 130(Pt 5):1194-205.

13. Saadoun $S$, Waters $P$, Bell BA, Vincent $A$, Verkman AS, Papadopoulos MC Intra-cerebral injection of neuromyelitis optica immunoglobulin $\mathrm{G}$ and human complement produces neuromyelitis optica lesions in mice. Brain. 2010;133:349-61.

14. Asavapanumas N, Ratelade J, Papadopoulos MS, Bennett JL, Levin MH, Verkman AS. Experimental mouse model of optic neuritis with inflammatory demyelination produced by passive transfer of neuromyelitis opticaimmoglobulin G. J Neuroinflamm. 2014;11:16.

15. Zhang H, Verkman AS. Longitudinally extensive NMO spinal cord pathology produced by passive transfer of NMO-lgG in mice lacking complement inhibitor CD59. J Autoimmun. 2014;53:67-77.

16. Pittock SJ, et al. Eculizumab in AQP4-lgG-positive relapsing neuromyelitis optica spectrum disorders: an open-label pilot study. Lancet Neurol. 2013; 12(6):554-62.

17. Yao X, Verkman AS. Marked central nervous system pathology in CD59 knockout rats following passive transfer of neuromyelitis optica immunoglobulin G. Acta Neuropath Commun. 2017;5(1):15.

18. Yao X, Verkman AS. Complement regulator CD59 prevents peripheral organ injury in rats made seropositive for neuromyelitis optica immunoglobulin G. Acta Neuropath Commun. 2017:5(1):57.

19. Svehag SE. Adverse effect of clinical intervention on the complement system. Complement Inflam. 1991;8(5-6):359-69.

20. Ballanti E, Perricone C, Greco E, Ballanti M, Muzio GD, Chimenti MS. Complement and autoimmunity. Immunol Res. 2013;56(2-3):477-91.

21. Wang Z, et al. Low expression of complement inhibitory protein CD59 contributes to humerol autoimmunity against astrocyte. Brain Behav Immun. 2017;65:173-82.

22. Bennett $J$, et al. Intrathecal pathogenic anti-aquaporin-4 antibodies in early neuromyelitis optica. Ann Neurol. 2009;66(5):617-29.

23. Crane JM, Lam C, Rossi A, Gupta T, Bennett JL, Verkman AS. Binding affinity and specificity of neuromyelitis optica autoantibodies to aquaporin-4 M1/ M23 isoforms and orthogonal arrays. J Biol Chem. 2011;286(18):16516-24.

24. Li L, Zhang H, Varrin-Doyer M, Zamvil SS, Verkman AS. Proinflammatory role of aquaporin-4 in autoimmune neuroinflammation. FASEB J. 2011; 25:556-1566.

25. Phuan PW, et al. C1q-targeted monoclonal antibody prevents complementdependent cytotoxicity and neuropathology in in vitro and mouse models of neuromyelitis optica. Acta Neuropathol. 2013;125(6):829-40.

26. Yao X, Su T, Verkman AS. Clobetasol promotes remyelination in a mouse model of neuromyelitis optica. Acta Neuropath Commun. 2016:4(1):42.

27. Ratelade J, Bennett JL, Verkman AS. Intravenous neuromyelitis optica autoantibody in mice targets aquaporin-4 in peripheral organs and area postrema. PLoS One. 2011;6(11):e27412.

28. Junnikkala S, Jokiranta TS, Friese MA, Jarva H, Zipfel PF, Meri S. Exceptional resistance of human $\mathrm{H} 2$ glioblastoma cells to complementmediated killing by expression and utilization of factor $\mathrm{H}$ and factor $\mathrm{H}$ like protein 1. J Immunol. 2000;164(11):6075-81.

29. Saheki A, Terasaki T, Tamai I, Tsuji A. In vivo and in vitro blood-brain barrier transport of 3-hydroxy-3-methylglutaryl coenzyme a (HMG-CoA) reductase inhibitors. Pharm Res. 1994;1 1(2):305-11.

30. Most JVD, Dolga AM, Nijholt IM, Luiten PGM, Eisel ULM. Statin: mechanism of neuroprotection. Prog Neurobiol. 2009;88(1):64-75.

31. Greenwood J, Steinman L, Zamvil SS. Statin therapy and autoimmune disease: from protein prenylation to immunomodulation. Nat Rev Immunol. 2006;6(5):358-70.

32. Zeiser R. Immune modulatory effects of statins. Immunology. 2018; 154(1):69-75

33. Youssef S, et al. The HMG-CoA reductase inhibitor, atorvastatin, promotes a Th2 bias and reverses paralysis in central nervous system autoimmune disease. Nature. 2002:420(6911):78-84.

34. Pannu R, Christie DK, Barbosa E, Singh I, Singh AK. Post-trauma Lipitor treatment prevents endothelial dysfunction, facilitates neuroprotection, and promotes locomotor recovery following spincal cord injury. J Neurochem. 2007:101(1):182-200

35. Xu X, et al. Anti-inflammatory and immunomodulatory mechanisms of atorvastatin in a murine model of traumatic brain injury. J Neuroinflamm. 2017: 4(1):167. 
36. Ratelade J, Asavapanumas N, Ritchie AM, Wemlinger S, Bennett JL, Verkman AS. Involvement of antibody-dependent cell-mediated cytotoxicity in inflammatory demyelination in a mouse model of neuromyelitis optica. Acta Neuropathol. 2013;126:699-709.

37. Laranjeira S, Symmonds M, Palace J, Payne SJ, Oriowski P. A mathematical model of cellular swelling in neuromyelitis optica. J Theor Biol. 2017:433:39-48.

38. Tradtrantip L, Asavapanumas N, Phuan PW, Verkman AS. Potential therapeutic benefit of C1-estrease inhibitor in neuromyelitis optica evaluated in vitro and in an experimental rat model. PLoS One. 2014;9(9): e106824.

39. Mason JC, Lidington EA, Yarwood H, Lublin DM, Haskard DO. Induction of endothelial cell decay-accelerating factor by vascular endothelial growth factor. Arthritis Rheumatol. 2001;44(1):138-50.

40. Mason JC, Lidington EA, Ahmad SR, Haskard DO. bFGF and VEGF synergistically enhance endothelial cytoprotection via decay-accelerating factor induction. Am J Physiol Cell Physiol. 2002;282(3):C578-87.

41. Mason JC, et al. Statin-induced expression of decay-accelerating factor protects vascular endothelium against complement-mediated injury. Circ Res. 2002;91(8):696-703.

42. Ahmad SR, et al. Decay-accelerating factor induction by tumour necrosis factor-a, through a phosphatidylinositol-3 kinase and protein kinase Cdependent pathway, protects murine vascular endothelial cells against complement deposition. Immunology. 2003;110(2):258-68.

43. Kinderlerer AR, et al. Statin-induced expression of CD59 on vascular endothelium in hypoxia: a potential mechanism for the anti-inflammatory actions of statins in rheumatoid arthritis. Arthritis Res \& Ther. 2006;8(4):R130.

44. Beckman JA, Creager MA. The nonlipid effects of statins on endothelial function. Trends Cardiovasc Med. 2006;16(5):156-62.

45. Greenwood J, Mason JC. Statins and the vascular endothelial inflammatory response. Trends Immunol. 2007;28(2):88-98.

46. Endres M, Laufs U. Effects of statins on endothelium and signaling mechanism. Stroke. 2004;35(11 Suppl 1):2708-11.

47. Sjogren AK, et al. GGTase-I deficiency reduces tumor formation and improves survival in mice with K-RAS-induced lung cancer. J Clin Invest. 2007;117(5):294-1304.

48. Sane $\mathrm{KM}$, et al. A novel geranylgeranyl transferase inhibitor in combination with lovastatin inhibits proliferation and induces autoghagy in STS-26T MPNST cells. J Pharmacol Exp Ther. 2010;333(1):23-33.

49. Zhou X, et al. Geranylgeranyltransferase I promotes human glioma cell growth through Rac1 membrane association and activation. J Mol Neurosci. 2013:49(1):130-9.

50. Bredel M, Pollack IF, Freund JM, Hamilton AD, Sebti SM. Inhibition of Ras and related G-proteins as a therapeutic strategy for blocking malignant glioma growth. Neurosurg. 1998;43(1):124-31.

51. Lu J, Chan L, Fiji HD, Dahl R, Kwon O, Tamanoi F. In vivo antitumor effect of a novel inhibitor of protein geranylgeranyltransferase-I. Mol Cancer Ther. 2009:8(5):1218-26.

52. O'Dwyer PJ, Gallagher M, Nguyen B, Waddell MJ, Chiorean EG. Phase I accelerated dose-escalating safety and pharmacokinetic (PK) study of GGTI2418, a novel geranylgeranyltransferase I inhibitor in patients with refractory solid tumors. Ann Oncol. 2010;21:ii42

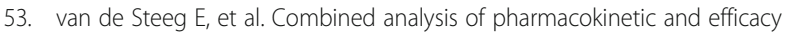
data of preclinical studies with statins markedly improves translation of drug efficacy to human trials. J Pharmacol Exp Ther. 2013;347(3):635-44.

54. Piermartiri TC, et al. Atorvastain prevents hippocampus cell death, neuroinflammation and oxidative stress following amyloid- $\beta(1-40)$ administration in mice: evidence for dissociation between cognitive deficits and neuronal damage. Exper Neurol. 2010;226:274-84.

55. Kurata T, et al. Atorvastatin and pitavastatin improve cognitive function and reduce senile plague and phosphorylated tau in aged APP mice. Brain Res. 2011;1371:161-70.

56. Saadoun S, Papadopoulos MC. Role of membrane complement regulators in neuromyelitis optica. Mult Scler. 2015;21:644-1654.

57. Klopfleisch $\mathrm{S}$, et al. Negative impact of statins on oligodendrocytes and myelin formation in vitro and in vivo. J Neurosci. 2008;28(50):13609-14.

58. Miron VE, et al. Statin therapy inhibits remyelination in the central nervous system. Am J Pathol. 2009;174(5):1880-90.

59. Pihl-Jensen G, Tsakiri A, Frederiksen JL. Statin treatment in multiple sclerosis: a systematic review and meta-analysis. CNS Drug. 2015;29:277-91.

\section{Ready to submit your research? Choose BMC and benefit from:}

- fast, convenient online submission

- thorough peer review by experienced researchers in your field

- rapid publication on acceptance

- support for research data, including large and complex data types

- gold Open Access which fosters wider collaboration and increased citations

- maximum visibility for your research: over $100 \mathrm{M}$ website views per year

At $\mathrm{BMC}$, research is always in progress.

Learn more biomedcentral.com/submissions 